\title{
High Power Microwaves Strategic and Operational Implications for Warfare
}



May 2000

Occasional Paper No. 11

Center for Strategy and Technology Air War College

Air University

Maxwell Air Force Base 